\title{
Six-month versus nine-month therapy for intestinal tuberculosis: a protocol for a randomized controlled study
}

\author{
Min Liu, Cheng Er Zhan, Linlin Shi, Li Zhao, Xia Jiang, Zhao Lin Zhang, Dongya Chen, Hong Xu, \\ Jianyong Chen
}

Affiliated Hangzhou Chest Hospital, Zhejiang University School of Medicine, Hangzhou, China

Correspondence to: Prof. Jianyong Chen. Affiliated Hangzhou Chest Hospital, Zhejiang University School of Medicine, 208 Huancheng East Road, Hangzhou, China. Email: 1lmy200611@163.com.

\begin{abstract}
Background: The optimal duration of treatment for intestinal tuberculosis (TB), which remains a common disease worldwide, has not yet been established. The proposed randomized controlled study will aim to compare the efficacy of short-term six-month with nine-month anti-TB therapy for treating intestinal TB.

Methods: This multicenter, open-label, double-blinded, randomized controlled trial conducted in the Affiliated Hangzhou Chest Hospital of Zhejiang University will include a total of 80 patients. Patients who meet the inclusion criteria will be randomly assigned to either the six-month $(n=40)$ or nine-month $(n=40)$ treatment group. The primary outcome will be complete response, which is defined as endoscopy displaying active lesion healing at the end of treatment. Participants will be scheduled for follow-up visits once a month in the first three months, then once every three months until the end of the treatment. The last follow-up will be one year after the treatment. Recurrence will be assessed one year after the end of treatment, which is defined as endoscopy displaying recurrent lesions after complete response.
\end{abstract}

Discussion: In addition to the reports of tuberculous lymphadenitis and spinal TB, there are few appropriate randomized trials for the treatment of extrapulmonary TB with appropriate clinical endpoints. We believe that the proposed randomized controlled trial will provide further data on the efficacy of shortterm six-month anti-TB therapy in intestinal TB patients.

Trial Registration: This trial will be registered on ClinicalTrial.gov.

Keywords: Intestinal tuberculosis (intestinal TB); short-term therapy; randomized controlled trial; complete response

Submitted Jun 04, 2021. Accepted for publication Jul 13, 2021.

doi: 10.21037/apm-21-1642

View this article at: https://dx.doi.org/10.21037/apm-21-1642

\section{Introduction}

Tuberculosis (TB) can occur in almost any organ or tissue of the body, and the range of clinical diseases is very wide. This disease has been successfully controlled since the introduction of effective anti-TB treatments and the improvement in people's living standards. However, in recent decades, there has been a trend towards resurgence of TB worldwide, and the global infection rate was estimated to be more than $30 \%$ according to the tuberculin surveys in 1997. The main reasons include the prevalence of human immunodeficiency virus (HIV) infection in some developing and developed countries. Moreover, the appearance of multidrug-resistant Mycobacterium tuberculosis has posed an increasing challenge to the successful control of TB in several parts of the world (1-3). TB is still a major problem in Africa and Asia, and continues to induce considerable morbidity and mortality in these regions (4). Extrapulmonary TB is becoming more and more common: the incidence of extrapulmonary organ infection was estimated to be between $15 \%$ and $20 \%$ in patients who are not infected by HIV, however, in those who are infected with TB and HIV concurrently, the incidence was reported to be between $50 \%$ and $70 \%(3,5,6)$. 
Among the extrapulmonary TB infections, intestinal TB is one of the most common diseases. The intestinal organs that are mainly involved include the distal ileum and cecum, followed by the jejunum, ileum, colon, and rectum $(5,7,8)$. Intestinal TB has been prevalent and has been a major health problem in developing countries. Combination chemotherapy with isoniazid, rifampicin, pyrazinamide, and ethambutol for six months has been a common and effective therapy for treating pulmonary TB. Adding pyrazinamide to this therapy containing isoniazid and rifampicin can shorten the treatment duration from nine months to six months (9). Although studies that have investigated extrapulmonary TB therapies are limited, there are reports that the basic principles of TB treatment can also be applied to extrapulmonary TB infections $(5,10-12)$. However, due to the difficulties of assessing bacteriological diagnosis and treatment response, many doctors, especially those in developing countries, have been reluctant to use six-month treatment for extrapulmonary $\mathrm{TB}$, such as intestinal TB.

Few randomized controlled studies investigating the optimal treatment duration of intestinal TB have been performed, except for several retrospective reviews $(13,14)$. Park et al. initiated a similar trial in 2009, however, it is not of double-blind design (15). Therefore, we aim to conduct this randomized, double-blind, clinical trial to compare the effect of six-month versus nine-month therapy in intestinal TB patients. We present the following article in accordance with the SPIRIT reporting checklist (16) (available at https://dx.doi.org/10.21037/apm-21-1642).

\section{Methods}

\section{Study design}

This trial will be a multicenter, open-label, double-blinded, randomized controlled trial conducted in Zhejiang, China. Patients with a clear diagnosis of intestinal TB will be eligible for the trial, and intestinal TB can be definitely diagnosed if at least one of the following criteria is met: (I) endoscopic biopsy shows caseous granuloma; (II) the histological specimen identifies the acid-fast bacilli; (III) Mycobacterium tuberculosis is positive in biopsy specimens; (IV) typical colonoscopy results strongly suggest that intestinal $\mathrm{TB}$ is associated with active pulmonary TB, regardless of acid-fast bacilli smear or sputum mycobacterium culture. The exclusion criteria will include: (I) age less than 18 years or more than 80 years; (II) infected with other extrapulmonary TB but not intestinal
TB; (III) received anti-TB chemotherapy in the past three years; and (IV) with concurrent chronic liver disease or immunosuppressive disorder. Patients who are pregnant will also be excluded in this trial for security reasons. The study design has been approved by the Review Board of the Affiliated Hangzhou Chest Hospital of Zhejiang University, and all the enrolled patients will be asked to give their informed consent.

\section{Pre-treatment evaluation and follow-up}

At the beginning of the trial, all patients will provide their medical and family history, then they will all receive a chest X-ray, blood counts, routine biochemical tests, and a complete physical examination. All patients will receive a colonoscopy both at the beginning of the trial and at the end of the anti-TB therapy. Patients will be scheduled for follow-up visits once a month in the first three months, then once every three months until the end of the treatment. The last follow-up will be one year after the treatment.

\section{Interventions and control}

The flow diagram of the proposed randomized controlled trial is showed in Figure 1. Patients with intestinal TB who meet the inclusion criteria will be randomly divided into two groups and treated with chemotherapy for either six months or nine months. A 1:1 simple randomization procedure will be used for the randomization based on a computergenerated randomization list. Only one external manager that is not involved in the trial will have the possession of the randomization list, and others including patients and care providers will all be blinded to the randomization. The six-month group of patients will receive the Z2H6R6E6 cocktail, which consists of rifampin, ethambutol, and isoniazid for six months, together with pyrazinamide for the first two months. Patients in the nine-month group will receive the Z2H9R9E9 combination, which is composed of rifampin, isoniazid, and ethambutol for nine months, together with pyrazinamide for the initial two months. Because of the high rate of primary drug resistance in South Korea (17), we will retain ethambutol for continued use. Isoniazid will be given at a dose of $300 \mathrm{mg} /$ day for patients under $50 \mathrm{~kg}$ of body weight and $400 \mathrm{mg} /$ day for those over $50 \mathrm{~kg}$. Rifampin will be given at a dose of $450 \mathrm{mg} /$ day for patients under $50 \mathrm{~kg}$ of body weight and $600 \mathrm{mg} /$ day for those over $50 \mathrm{~kg}$. The doses of ethambutol $(15-20 \mathrm{mg} / \mathrm{kg})$ and pyrazinamide $(20-30 \mathrm{mg} / \mathrm{kg})$ will be, respectively, 


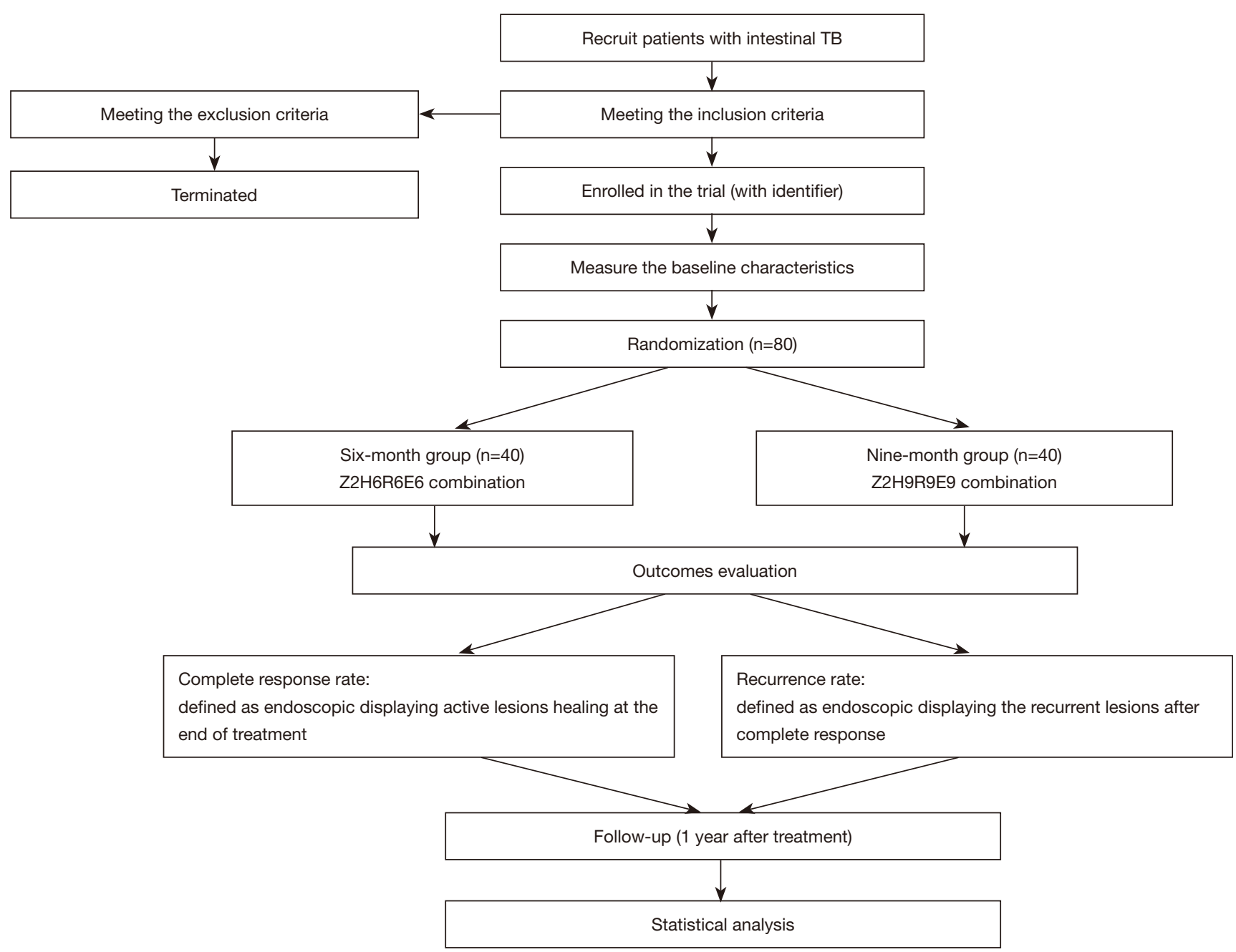

Figure 1 The flow diagram of the proposed randomized controlled trial. TB, tuberculosis; Z2H6R6E6, rifampin, ethambutol, and isoniazid for six months, together with pyrazinamide for the first two months; Z2H9R9E9, rifampin, isoniazid, and ethambutol for nine months, together with pyrazinamide for the initial two months.

$1,000 \mathrm{mg} /$ day and $1,250 \mathrm{mg} /$ day for those under $50 \mathrm{~kg}$ of body weight, and $1,200 \mathrm{mg} / \mathrm{day}$ and $1,500 \mathrm{mg} /$ day for those over $50 \mathrm{~kg}$. After the initial two months, the dose of ethambutol will be reduced to $800 \mathrm{mg}$ /day for all participants. Corticosteroids will not be used for any participants, while surgery will mainly be used to treat intestinal obstruction, perforation, fistula, and other complications (18).

\section{Discontinuation}

Discontinuation criteria will include serious adverse events, complications, and failure to continue the trial. Patients who cannot carry out the corresponding treatment or participate in the outcome assessment due to personal reasons will also be terminated. For these patients, the reason for discontinuation will be recorded clearly, and the outcomes will be recorded and evaluated in detail. Serious adverse events will be reported to the ethics committee immediately. No interim analysis will be performed in the proposed study.

\section{Outcomes}

The data of the two groups at diagnosis, treatment end, and one year after the treatment end will be compared to evaluate the improvement in abdominal symptoms and the rate of complete healing of active lesions in endoscopic recording. 
Complete response will be defined as endoscopy displaying active lesion healing at the end of treatment. Recurrence will be defined as endoscopy displaying recurrent lesions after complete response. Since most recurrences occur within the first six to twelve months after discontinuation of treatment $(9,19)$, we will not assess the disease status until one year after the end of treatment.

\section{Sample size}

A total of 80 patients (40 in each group) will be needed to realize the statistical power if the true success rate in the nine-month group is set as $95 \%$, and the possibility that the success rate in the six-month group is at least $5 \%$ less than that of the nine-month group (the noninferiority of the sixmonth group hypothesis; one-sided $\mathrm{P}<0.05$ ).

\section{Data collection and management}

Baseline characteristics of the enrolled patients will be collected by two authors independently. Data collected will include age, gender, weight, previous history of intestinal $\mathrm{TB}$, and corresponding treatment received. Double data entry will be used to promote data quality. All the data will be reviewed by the data monitoring committee, which is composed of five professional experts who are blinded to the trial process, so as to increase the accuracy of our data.

\section{Statistical analysis}

All outcomes will be compared on the basis of the intentionto-treat principle. The Wilcoxon rank sum test will be used to compare continuous variables between groups, while the chi-squared test or Fisher's exact test will be used for categorical variables, as appropriate. Subgroup analyses will be performed based on different age groups and gender. All $\mathrm{P}$ values will be two-sided, with $\mathrm{P}<0.05$ considered as statistically significant. Analyses will be performed using SPSS Software (version 21.0, SPSS Inc., Chicago, IL).

\section{Ethics}

Prior to the start of this study, we obtained approval of this protocol from the Review Board of the Affiliated Hangzhou Chest Hospital of Zhejiang University. Before the clinical trial, all participants will provide written informed consent to participate in the study. A clinician in our hospital who is blinded to the trial process will collect the written informed consent and the information collected will be strictly confidential. Any changes to the protocol will be reported to the Review Board immediately. All procedures performed in this study involving human participants will be in accordance with the Declaration of Helsinki (as revised in 2013).

\section{Discussion}

Based on the data of in vitro and animal experiments, clinicians have made efforts to shorten the treatment duration of pulmonary TB (20). In these studies, the combination of drugs that are effective in killing propagules (isoniazid and rifampicin) and drugs that attack intracellular organisms (pyrazinamide) was used to improve the overall efficacy of chemotherapy and allow the total duration of treatment to be shortened to six months. In our opinion, these data seem to be applicable to the treatment of extrapulmonary TB.

The treatment of intestinal tuberculosis depends on regular anti tuberculosis drug therapy and the improvement of life style. Our study aims to investigate whether sixmonth therapy is effective in treating intestinal TB. Up to now, there is growing data which suggests that therapies with isoniazid and rifampin are indeed effective for this disease. Therefore, a six-month duration of treatment is recommended for TB in any part of the body except the meninges, and in some cases, the bones, where nine- to twelve-month treatment may be applied instead. However, in addition to the reports of tuberculous lymphadenitis $(21,22)$ and spinal TB $(23-25)$, there are few appropriate randomized trials for the treatment of extrapulmonary $\mathrm{TB}$ with appropriate clinical endpoints. We believe that our trial will provide important data comparing the efficacy of short-term six-month therapy with longer-term nine-month therapy. The above steps will be implemented strictly to ensure the reliability of our results.

\section{Acknowledgments}

Funding: None.

\section{Footnote}

Reporting Checklist: The authors have completed the SPIRIT reporting checklist. Available at https://dx.doi. org/10.21037/apm-21-1642

Conflicts of Interest: All authors have completed the ICMJE 
uniform disclosure form (available at https://dx.doi. org/10.21037/apm-21-1642). The authors have no conflicts of interest to declare.

Ethical Statement: The authors are accountable for all aspects of the work in ensuring that questions related to the accuracy or integrity of any part of the work are appropriately investigated and resolved. All procedures performed in this study involving human participants will be in accordance with the Declaration of Helsinki (as revised in 2013). The study design has been approved by the Review Board of the Affiliated Hangzhou Chest Hospital of Zhejiang University, and all the enrolled patients will be asked to give their informed consent.

Open Access Statement: This is an Open Access article distributed in accordance with the Creative Commons Attribution-NonCommercial-NoDerivs 4.0 International License (CC BY-NC-ND 4.0), which permits the noncommercial replication and distribution of the article with the strict proviso that no changes or edits are made and the original work is properly cited (including links to both the formal publication through the relevant DOI and the license). See: https://creativecommons.org/licenses/by-nc-nd/4.0/.

\section{References}

1. Corbett EL, Watt CJ, Walker N, et al. The growing burden of tuberculosis: global trends and interactions with the HIV epidemic. Arch Intern Med 2003;163:1009-21.

2. Dye C, Watt CJ, Bleed DM, et al. Evolution of tuberculosis control and prospects for reducing tuberculosis incidence, prevalence, and deaths globally. JAMA 2005;293:2767-75.

3. Golden MP, Vikram HR. Extrapulmonary tuberculosis: an overview. Am Fam Physician 2005;72:1761-8.

4. Tuberculosis control and research strategies for the 1990s: memorandum from a WHO meeting. Bull World Health Organ 1992;70:17-21.

5. Marshall JB. Tuberculosis of the gastrointestinal tract and peritoneum. Am J Gastroenterol 1993;88:989-99.

6. Rieder HL, Snider DE Jr, Cauthen GM. Extrapulmonary tuberculosis in the United States. Am Rev Respir Dis 1990;141:347-51.

7. Farer LS, Lowell AM, Meador MP. Extrapulmonary tuberculosis in the United States. Am J Epidemiol 1979;109:205-17.

8. Horvath KD, Whelan RL. Intestinal tuberculosis: return of an old disease. Am J Gastroenterol 1998;93:692-6.

9. A controlled trial of six months chemotherapy in pulmonary tuberculosis. First Report: results during chemotherapy. British Thoracic Association. Br J Dis Chest 1981;75:141-53.

10. Cohn DL, Catlin BJ, Peterson KL, et al. A 62-dose, 6-month therapy for pulmonary and extrapulmonary tuberculosis. A twice-weekly, directly observed, and costeffective regimen. Ann Intern Med 1990;112:407-15.

11. Joint T. Chemotherapy and management of tuberculosis in the United Kingdom: recommendations 1998. Joint Tuberculosis Committee of the British Thoracic Society. Thorax 1998;53:536-48.

12. Small PM, Fujiwara PI. Management of tuberculosis in the United States. N Engl J Med 2001;345:189-200.

13. Bastani B, Shariatzadeh MR, Dehdashti F. Tuberculous peritonitis--report of 30 cases and review of the literature. Q J Med 1985;56:549-57.

14. Demir K, Okten A, Kaymakoglu S, et al. Tuberculous peritonitis--reports of 26 cases, detailing diagnostic and therapeutic problems. Eur J Gastroenterol Hepatol 2001;13:581-5.

15. Park SH, Yang SK, Yang DH, et al. Prospective randomized trial of six-month versus nine-month therapy for intestinal tuberculosis. Antimicrob Agents Chemother 2009;53:4167-71.

16. Chan AW, Tetzlaff JM, Altman DG, et al. SPIRIT 2013 statement: defining standard protocol items for clinical trials. Ann Intern Med 2013;158:200-7.

17. Seung KJ, Bai GH, Kim SJ, et al. The treatment of tuberculosis in South Korea. Int J Tuberc Lung Dis 2003;7:912-9.

18. Akgun Y. Intestinal and peritoneal tuberculosis: changing trends over 10 years and a review of 80 patients. Can J Surg 2005;48:131-6.

19. Mitchison DA. Role of individual drugs in the chemotherapy of tuberculosis. Int J Tuberc Lung Dis 2000;4:796-806.

20. Grosset J. Bacteriologic basis of short-course chemotherapy for tuberculosis. Clin Chest Med 1980;1:231-41.

21. Short course chemotherapy for tuberculosis of lymph nodes: a controlled trial. British Thoracic Society Research Committee. Br Med J (Clin Res Ed) 1985;290:1106-8.

22. Campbell IA, Ormerod LP, Friend JA, et al. Six months versus nine months chemotherapy for tuberculosis of lymph nodes: final results. Respir Med 1993;87:621-3.

23. Darbyshire J. Five-year assessment of controlled trials of 
short-course chemotherapy regimens of 6, 9 or 18 months' duration for spinal tuberculosis in patients ambulatory from the start or undergoing radical surgery. Fourteenth report of the Medical Research Council Working Party on Tuberculosis of the Spine. Int Orthop 1999;23:73-81.

24. Controlled trial of short-course regimens of chemotherapy in the ambulatory treatment of spinal tuberculosis. Results at three years of a study in Korea. Twelfth report of the
Medical Research Council Working Party on Tuberculosis of the Spine. J Bone Joint Surg Br 1993;75:240-8.

25. Parthasarathy R, Sriram K, Santha T, et al. Short-course chemotherapy for tuberculosis of the spine. A comparison between ambulant treatment and radical surgery--ten-year report. J Bone Joint Surg Br 1999;81:464-71.

(English Language Editor: C. Betlazar-Maseh)
Cite this article as: Liu M, Zhan CE, Shi L, Zhao L, Jiang X, Zhang ZL, Chen D, Xu H, Chen J. Six-month versus ninemonth therapy for intestinal tuberculosis: a protocol for a randomized controlled study. Ann Palliat Med 2021;10(8):92339238. doi: 10.21037/apm-21-1642 